\title{
ARTIGO ORIGINAL Transtornos mentais comuns entre os estudantes do curso de medicina: prevalência e fatores associados
}

Common mental disorders in medical students: prevalence and associated factors

Karoline Pedroti Fiorotti', Renzo Roldi Rossoni', Luiz Henrique Borges², Angélica Espinosa Miranda ${ }^{3}$

\section{RESUMO}

Objetivo: O objetivo do estudo foi estimar a prevalência de transtornos mentais comuns (TMC) entre os estudantes do curso de medicina da UFES e avaliar possíveis correlações entre TMC e fatores de risco. Métodos: Estudo transversal realizado de setembro a novembro de 2007 envolvendo 229 alunos do curso de medicina. O questionário utilizado foi autoaplicável e anônimo. Foram coletados dados socioeconômicos, informações sobre o curso, o processo de ensino-aprendizagem e a rede de apoio social. Para o rastreamento de TMC, utilizou-se o Self-Reporting Questionnaire (SRQ-20). Resultados: A prevalência total de TMC encontrada foi de 37,1\% (IC 95\%, 30,8\%-43,4\%), que esteve independentemente associada a não receber o apoio emocional necessário (OR =7,4, IC 95\%, 3,1-17,9) e relatar "dificuldade para tirar dúvidas em sala de aula por timidez" durante a infância ou adolescência (OR =2,5, IC 95\%, 1,0-6,1). Conclusão: Os dados demonstram elevada prevalência de TMC nessa população e a importância em subsidiar ações para prevenção e cuidado com a saúde mental dos estudantes, melhorando a qualidade de vida deles.

\section{ABSTRACT}

Objective: The goal of this study was to estimate the prevalence of Common Mental Disorders (CMD) in medical students from Universidade Federal do Espírito Santo and evaluate possible correlation among CMD and risk factors. Methods: A cross-sectional study performed from September to November 2007 enrolled 229 medical students. A self-applicable and anonymous questionnaire was used. Information on socioeconomic characteristics, about the course, teaching-learning process and support assistance were collected. CMD was screened by SRQ-20 questionnaire. Results: Global prevalence was 37.1\% (IC 95\%, 30.8\%-43.4\%) and it was independent correlated to not receiving sufficient emotional support (OR $=7.4,1 \mathrm{C} 95 \%, 3.1-17.9)$ and report "difficulties for asking questions during classes, by shyness" during childhood and adolescence (OR $=2.5$, IC 95\%, 1.0-6.1).

\section{Keywords}

Mental disorders, medical students, risk factors, prevalence, mental health. Conclusion: These data showed high prevalence of CMD in this population and the importance of planning prevention and assistance strategies regarding mental health focuses on a better quality of life for these students.

1 Universidade Federal do Espírito Santo (UFES).

2 Santa Casa de Misericórdia de Vitória, Escola de Medicina, Departamento de Saúde Coletiva. 


\section{INTRODUÇÃO}

Os transtornos mentais comuns (TMC), também conhecidos como transtornos psiquiátricos menores, representam os quadros menos graves e mais frequentes de transtorno mental'. Os sintomas de TMC incluem esquecimento, dificuldade na concentração e tomada de decisões, insônia, irritabilidade e fadiga, assim como queixas somáticas (cefaleia, falta de apetite, tremores, má digestão, entre outros), mas não incluem transtornos psicóticos, dependência química ou transtornos de personalidade². Ser portador de TMC é uma condição que não implica diagnóstico psiquiátrico formal, porém representa custos enormes em termos de sofrimento psíquico e impacto nos relacionamentos e na qualidade de vida, comprometendo o desempenho nas atividades diárias e constituindo causa importante de afastamento do trabaIho, demanda nos serviços de saúde e prejuízos econômicos, sendo potencial substrato para o desenvolvimento de transtornos mais graves ${ }^{3-6}$.

O ingresso do estudante no ambiente universitário consiste em múltiplos processos que envolvem aspectos externos, dos ambientes acadêmico e social, e aspectos internos do indivíduo, como a habilidade de encarar as diversas situações, as reações físicas psicossomáticas e os diferentes estados de humor ${ }^{7}$. Muitos indivíduos irão apresentar o seu primeiro episódio psiquiátrico durante a graduação e, segundo uma revisão, 12\% a 18\% dos universitários apresentam alguma doença mental diagnosticável| ${ }^{8}$. Alguns estudos conduzidos na área da saúde mental têm sido focados em estudantes da área da saúde ${ }^{7,9}$, principalmente do curso de medicina, que expõe os estudantes a fontes de tensão desde o processo de admissão até o final da graduação, com a entrada no mercado de trabalho e em programas de residência médica ${ }^{10,11}$.

Os fatores de estresse, presentes na graduação médica, incluem competição no processo de seleção, sobrecarga de conhecimentos, dificuldade na administração do tempo entre um grande número de atividades e pouco tempo para atividades de lazer, individualismo, responsabilidade e expectativas sociais do papel do médico. Outros fatores incluem o contato com a morte e inúmeros processos patológicos, o exame físico em um paciente, o medo de adquirir doenças, o medo de cometer erros e sentimentos de impotência diante de certas doenças ${ }^{10,11}$. Tudo isso pode levar os estudantes a acionarem mecanismos de defesa psicológicos tais como dissociação ou isolamento afetivo?.

Estudos da literatura científica demonstram alta prevalência de desordens psíquicas nessa população, um estudo africano demonstrou que $64,5 \%$ dos estudantes de medicina do primeiro período apresentavam algum grau de depressão, com $11 \%$ demonstrando altíssimos níveis de estresse ${ }^{12}$. Em um estudo conduzido em nove escolas médicas nos Estados Unidos, 46\% dos estudantes apresentavam pelo menos um dos sintomas investigados - estresse, fadiga, ansiedade, depressão, cefaleia e distúrbios alimentares ${ }^{13}$. No Brasil, um estudo conduzido na Universidade Federal de Santa Maria usando Self-Reporting Questionnaire (SRQ-20) mostrou que a prevalência de TMC entre os estudantes do primeiro ao décimo semestre do curso de medicina foi de 31,7\%"11.

Um estudo longitudinal publicado em 1997 demonstrou um aumento progressivo nos escores de depressão dos estudantes durante a escola médica, o que sugere que o sofrimento mental para essa população é crônico e persistente ${ }^{14}$. Dados sobre TMC são importantes para subsidiar ações para prevenção e cuidado com a saúde mental dessa população, melhorando a qualidade de vida dela e, consequentemente, auxiliando em sua formação profissional. Além disso, seriam importantes para colaborar com reflexões sobre o modelo curricular vigente nas escolas médicas.

O objetivo deste estudo foi identificar a prevalência de TMC entre estudantes do curso de medicina da Universidade Federal do Espírito Santo (UFES) e determinar a correlação entre TMC e possíveis fatores preditivos.

\section{MÉTODOS}

\section{População de estudo}

Foi realizado um estudo transversal com 240 alunos do primeiro ao sexto ano do curso de medicina da UFES, entre setembro e novembro de 2007, selecionados por meio da lista de matrícula fornecida pelo colegiado de curso.

\section{Seleção da amostra e coleta de dados}

O curso de medicina da UFES possui 480 alunos regularmente matriculados, sendo 40 alunos em cada período acadêmico. Foram selecionados os primeiros 20 alunos de numeração ímpar, do $1^{\circ}$ ao $11^{\circ}$ período, em cada lista oficial de matrícula fornecida pelo colegiado de curso, totalizando 220 alunos. Quando o aluno selecionado não estava matriculado na turma informada pelo colegiado, o critério utilizado foi selecionar o aluno inscrito imediatamente acima na lista de matrícula. Quando a quantidade de alunos de numeração ímpar, matriculados num período, não alcançava o desejado, eram selecionados os primeiros alunos de numeração par, até completar a quantidade desejada. Quanto aos alunos do $12^{\circ}$ período, que estavam cursando o internato optativo, por causa da dificuldade de encontrá-los nas dependências da universidade, optouse por não utilizar critérios de amostragem, e, sim, abordar os alunos que eventualmente fossem encontrados ao longo da coleta de dados, objetivando, assim como nos outros períodos, o número ideal de 20 questionários entregues. A meta final, portanto, era de 240 alunos, sendo 20 alunos em cada período, do primeiro ao sexto ano do curso de medicina da UFES.

O tamanho da amostra foi calculado para estimar a taxa de prevalência de TMC em estudantes de medicina da UFES, 
com um intervalo de confiança de $95 \%$. Tomou-se como base para cálculo uma taxa média de prevalência de 35\%16,17, com variação de 5\%, o que gerou um número de 202 alunos. Considerando uma possível perda de aproximadamente 20\%, o tamanho de amostra final necessário foi de 240 estudantes.

\section{Instrumento de coleta}

Os questionários utilizados na pesquisa foram autoaplicáveis e anônimos. O primeiro questionário foi baseado nas informações coletadas nos estudos brasileiros anteriores e continha dados socioeconômicos (período, idade, sexo, cor, estado civil, procedência, religião, renda, escolaridade dos pais, instituições de ensino fundamental e médio), informações sobre o curso (motivo de escolha, satisfação, desejo de abandonar o curso, desempenho, reprovação, perspectivas, carga horária, atividades extracurriculares, sobrecarga, horas livres e atividades exercidas, falta às aulas), sobre o processo de ensino-aprendizagem (vestibulares prestados, relação professor-aluno, estratégias de ensino no curso, fontes de estudo, fontes de tensão), sobre a rede de apoio (relacionamento dos pais, confidente, grupo de amigos, rejeição, apoio emocional), história de tratamento medicamentoso ou psicoterapêutico e situações especiais durante a infância e/ou adolescência (doença, reprovação, timidez, medos, dificuldade para dormir, dificuldade para fazer amigos, desconforto físico, problema com autoridades, problemas de relacionamento com os pais, mau comportamento na escola, desinteresse por relacionamentos afetivos, dificuldade para conciliar estudos com lazer).

Para o rastreamento de TMC, utilizou-se um segundo questionário, o Self-Reporting Questionnaire (SRQ-20), instrumento desenvolvido para rastrear distúrbios psiquiátricos em centros de atenção primária à saúde ${ }^{15}$, validado no Brasil ${ }^{16}$ e recomendado pela Organização Mundial da Saúde ${ }^{17}$. Esse instrumento é composto por 20 perguntas com respostas "sim" ou "não", sendo 4 perguntas sobre sintomas físicos e 16 sobre desordens psicoemocionais. Os sintomas avaliados são referentes aos últimos 30 dias, e a cada resposta "sim" é atribuído um ponto, resultando em uma pontuação final que varia de 0 a 20 pontos. Os alunos foram distribuídos em dois grupos, de acordo com a pontuação no SRQ-20, pois o ponto de corte é diferente. Homens com pontuação menor ou igual a cinco e mulheres com pontuação menor ou igual a sete foram classificados como "não suspeitos". Homens com pontuação maior ou igual a seis e mulheres com pontuação maior ou igual a oito foram classificados como "suspeitos"15.

Os alunos foram contatados em sala de aula, na universidade, onde leram e assinaram o termo de consentimento e receberam os questionários para serem respondidos. Eles foram orientados a depositar o questionário em uma urna colocada em local de fácil acesso no diretório acadêmico do curso. O aluno selecionado que não foi encontrado ou que não devolveu o questionário foi considerado como "perda".

\section{Análise de dados}

Para a análise dos dados, foi utilizado o programa SPSS Inc 10.0. Foi criada uma variável TMC a partir do escore total de cada indivíduo no SRQ-20, no qual os sujeitos foram classificados como "suspeitos" de TMC ou não, de acordo com os pontos de corte, já especificados anteriormente.

Inicialmente, foi realizada uma análise descritiva da população estudada segundo as variáveis em estudo (presença de TMC, variáveis sociodemográficas, referentes ao curso, ao processo de ensino-aprendizagem e à rede de apoio social). Para análise dos dados, as variáveis categóricas foram representadas pelas suas frequências absolutas. Ao considerar a variável de desfecho (presença ou ausência de TMC) e as possíveis exposições (presença ou ausência de cada fator preditivo), a análise bivariada trabalhou com uma resposta do tipo dicotômica com comparação de dois grupos. O teste estatístico utilizado foi o qui-quadrado, exceto quando frequências menores do que cinco foram encontradas. Nessa situação, foi utilizado o teste exato de Fisher. A determinação das medidas de efeito foi feita por meio da razão das chances (odds ratio). As análises multivariadas foram conduzidas por meio de regressão logística. Foram incluídas no modelo final de regressão variáveis que foram estatisticamente significativas $(p<0,05)$ e/ou que se mostraram significativas em outros estudos, como sexo.

\section{Aspectos éticos}

O presente trabalho foi submetido ao Comitê de Ética em Pesquisa da Universidade Federal do Espírito Santo e aprovado em setembro de 2007. Todos os alunos foram informados sobre os objetivos da pesquisa e sobre o anonimato do questionário, assinando um Termo de Consentimento Livre e Esclarecido quando optaram pela participação.

\section{RESULTADOS}

Dentre os 240 questionários entregues, obteve-se retorno de 229 questionários (95,4\% da amostra selecionada), representados por $78(34,1 \%)$ alunos do curso básico (do 10 ao $4^{\circ}$ período), 77 (33,6\%) do curso clínico (do 50 ao 8 o período) e 74 (32,3\%) do internato (do $9^{\circ}$ ao $12^{\circ}$ período). As perdas foram homogêneas entre as turmas, perfazendo no máximo $10 \%$ de perda da amostra em cada período.

Em relação à saúde mental, 85 estudantes $[37,1 \%$ (IC 95\%, 30,8\%-43,4\%)] obtiveram pontuação que os classifica como casos "suspeitos" de TMC. O estudo apontou maior prevalência de TMC entre os alunos do curso básico $(43,6 \%)$ e do clínico (40,3\%), sendo menor no internato (27\%) $(p=$ $0,025)$. Estratificando os resultados, notou-se que as maiores prevalências foram encontradas no segundo $(52,6 \%)$ e no quarto $(53,8 \%)$ anos do curso de medicina da UFES; e a me- 
nor prevalência foi encontrada no último ano, com apenas $16,2 \%(p=0,008)$.

Foi encontrada maior prevalência de TMC entre as muIheres (40\%), indivíduos de crença espírita (66,7\%), que não possuem renda própria (43\%), com renda familiar de até $\mathrm{R} \$ 1.500,00(41,7 \%)$, que não estão satisfeitos com a escoIha profissional (100\%), que pensam em abandonar o curso (50\%) e que consideram seu desempenho acadêmico insuficiente $(66,7 \%)$. Observou-se também maior prevalência de TMC entre aqueles alunos que relatam dificuldade para fazer amigos (53,6\%), sentimento de rejeição por amigos ou outros da mesma faixa etária $(62,5 \%)$, não recebimento do apoio emocional necessário (64\%), história de tratamento psiquiátrico medicamentoso $(54,3 \%)$ e história de tratamento psicoterapêutico $(47,9 \%)$.

A tabela 1 apresenta dados sobre os aspectos sociais e econômicos. Ter entre 20 e 23 anos [OR = 2,5 (IC 95\%, 1,23$5,25)]$, ser espírita [OR =1,9 (IC 95\%, 1,22-2,94)] e não possuir renda própria [OR = 1,6 (IC 95\%, 1,04-2,30)] foram fatores que mostraram associação com TMC na análise bivariada.

Tabela 1. Dados sociodemográficos e associação com casos suspeitos de transtornos mentais comuns entre estudantes de medicina da UFES, $2007(\mathrm{~N}=229)$

\begin{tabular}{|c|c|c|c|c|c|}
\hline Variáveis & N total & $\%$ & $\%$ TMC & $p$ & $O R_{b}(I C 95 \%)$ \\
\hline Idade & & & & 0,032 & \\
\hline Até 19 anos & 36 & 15,7 & 33,3 & & $1,7(0,65-4,30)$ \\
\hline 20 a 23 anos & 141 & 61,6 & 43,3 & & $2,5(1,23-5,25)$ \\
\hline 24 anos ou mais & 52 & 22,7 & 23,1 & & 1 \\
\hline Sexo & & & & 0,365 & \\
\hline Feminino & 114 & 49,8 & 40,0 & & $1,2(0,83-1,64)$ \\
\hline Masculino & 115 & 50,2 & 34,2 & & 1 \\
\hline Religiäo & & & & 0,035 & \\
\hline Espírita & 12 & 5,2 & 66,7 & & $1,9(1,22-2,94)$ \\
\hline Outras/Não tem & 216 & 94,3 & 35,2 & & 1 \\
\hline Renda própria & & & & 0,023 & \\
\hline Não possui renda própria & 142 & 62 & 43,0 & & $1,6(1,04-2,30)$ \\
\hline $\begin{array}{l}\text { Sim, possui alguma } \\
\text { renda própria }\end{array}$ & 83 & 36,2 & 27,7 & & 1 \\
\hline Renda familiar & & & & 0,905 & \\
\hline Até $R \$ 1.500,00$ & 12 & 5,2 & 41,7 & & $1,5(0,44-4,88)$ \\
\hline $\begin{array}{l}\text { Entre } R \$ 1.500,00 \mathrm{e} \\
\mathrm{R} \$ 10.000,00\end{array}$ & 175 & 76,4 & 37,7 & & $1,6(0,91-2,79)$ \\
\hline Acima de $R \$ 10.000,00$ & 40 & 17,5 & 35,0 & & 1 \\
\hline Escolaridade do pai & & & & 0,082 & \\
\hline Até $2^{\circ} \mathrm{grau}$ & 91 & 39,7 & 44,0 & & $1,4(0,97-1,88)$ \\
\hline Ensino superior ou mais & 138 & 60,3 & 32,6 & & 1 \\
\hline Escolaridade da mãe & & & & 0,073 & \\
\hline Até $2^{\circ} \mathrm{grau}$ & 75 & 32,8 & 45,3 & & $1,4(0,98-1,91)$ \\
\hline Ensino superior ou mais & 153 & 66,8 & 33,1 & & \\
\hline
\end{tabular}

OR (IC 95\%): odds ratio bruto (intervalo de confiança de 95\%). Foram aplicados os testes de qui-quadrado ou Fisher quando apropriado.
Na tabela 2 estão descritas as informações sobre o curso e o processo de ensino-aprendizagem. Não estar satisfeito com a escolha profissional [OR =2,8 (IC 95\%, 2,36-3,37)], excesso de carga horária como fonte de tensão [OR = 1,7 (IC 95\%, 1,042,76)], não praticar atividades de lazer na frequência desejada $[\mathrm{OR}=2,4$ (IC 95\%, 1,13-5,04)] e sobrecarga de atividades por diversos motivos, como a busca de novos aprendizados e experiências $[\mathrm{OR}=1,6$ (IC 95\%, 1,5-2,24)], a cobrança pessoal $[O R=1,5($ IC $95 \%, 1,10-2,14)]$ e a pressão dos professores ou profissionais da área [OR = 1,8 (IC 95\%, 1,24-2,46)] foram as variáveis importantes para a apresentação de TMC.

Tabela 2. Dados relacionados ao curso e associação com casos suspeitos de transtornos mentais comuns entre os estudantes de medicina da UFES, $2007(\mathrm{~N}=229)$

\begin{tabular}{|c|c|c|c|c|c|}
\hline Variáveis & $\mathrm{N}$ & $\%$ & \% TMC & $\mathrm{p}$ & $\mathrm{OR}_{\mathrm{b}}(\mathrm{IC} 95 \%)$ \\
\hline Satisfação com a escolha profissional & & & & 0,001 & \\
\hline Não & 7 & 3,1 & 100,0 & & $2,8(2,36-3,37)$ \\
\hline Sim & 220 & 96,1 & 35,5 & & 1 \\
\hline Já pensou em abandonar o curso & & & & 0,544 & \\
\hline Sim, ainda penso & 16 & 7,0 & 50,0 & & $1,8(0,63-5,07)$ \\
\hline Sim, mas não penso mais & 81 & 35,4 & 37,0 & & $1,1(0,59-1,87)$ \\
\hline Não, nunca & 131 & 57,2 & 35,9 & & 1 \\
\hline Desempenho acadêmico & & & & 0,344 & \\
\hline Insuficiente & 6 & 2,6 & 66,7 & & $4,0(0,54-29,81)$ \\
\hline Regular & 55 & 24,0 & 41,8 & & $1,4(0,43-4,77)$ \\
\hline Bom & 151 & 65,9 & 34,4 & & $1,1(0,34-3,24)$ \\
\hline Excelente & 15 & 6,6 & 33,3 & & 1 \\
\hline $\begin{array}{l}\text { Contribuiç̧ão de carga horária de atividades } \\
\text { como fonte de tensão no curso }\end{array}$ & & & & 0,022 & \\
\hline Contribui muito & 171 & 74,7 & 41,5 & & $1,7(1,04-2,76)$ \\
\hline Contribui pouco & 57 & 24,9 & 24,6 & & 1 \\
\hline \multicolumn{6}{|l|}{ Atividades extracurriculares } \\
\hline Não praticar esporte & 195 & 85,2 & 40,0 & 0,031 & $1,9(0,98-3,84)$ \\
\hline Não estar envolvido com música & 201 & 87,8 & 39,8 & 0,024 & $2,2(0,99-5,02)$ \\
\hline \multicolumn{6}{|l|}{ Motivo da sobrecarga de atividades } \\
\hline \multicolumn{6}{|l|}{ Carga horária curricular } \\
\hline Muito extensa & 142 & 62,0 & 42,3 & 0,046 & $1,5(0,99-2,13)$ \\
\hline Normal & 87 & 38,0 & 29,1 & & 1 \\
\hline \multicolumn{6}{|l|}{$\begin{array}{l}\text { Busca de novos aprendizados } \\
\text { e experiências }\end{array}$} \\
\hline Sim & 94 & 41,0 & 47,9 & 0,006 & $1,6(1,15-2,24)$ \\
\hline Não & 135 & 59,0 & 29,9 & & 1 \\
\hline \multicolumn{6}{|l|}{ Cobrança pessoal } \\
\hline Sim & 117 & 51,1 & 43,6 & 0,043 & $1,4(1,01-2,02)$ \\
\hline Não & 112 & 48,9 & 30,6 & & 1 \\
\hline \multicolumn{6}{|l|}{ Pressão social da profissão } \\
\hline $\operatorname{sim}$ & 62 & 27,1 & 50,0 & 0,015 & $1,5(1,10-2,14)$ \\
\hline Não & 167 & 72,9 & 32,5 & & 1 \\
\hline \multicolumn{6}{|l|}{$\begin{array}{l}\text { Pressão dos professores ou profissionais } \\
\text { da área }\end{array}$} \\
\hline $\operatorname{Sim}$ & 36 & 15,7 & 58,3 & 0,004 & $1,8(1,24-2,46)$ \\
\hline Não & 193 & 84,3 & 33,3 & & 1 \\
\hline \multicolumn{6}{|l|}{$\begin{array}{l}\text { Praticar atividades de lazer na frequência } \\
\text { desejada }\end{array}$} \\
\hline Sim & 36 & 15,7 & 17,1 & 0,007 & 1 \\
\hline Não & 193 & 84,3 & 40,9 & & $2,4(1,13-5,04)$ \\
\hline
\end{tabular}

OR (IC 95\%): odds ratio bruto (intervalo de confiança de $95 \%$ ). Foram aplicados os testes de qui-quadrado ou Fisher quando apropriado. 
O relato de ter dificuldade para fazer amigos $(p=0,003)$, sentimento de rejeição por amigos $(p=0,029)$, não receber apoio emocional necessário $(p=0,001)$, história de tratamento psiquiátrico medicamentoso $(p=0,020)$ e história de tratamento psicoterapêutico $(p=0,020)$ foram fatores associados com TMC na análise bivariada. As situações especiais identificadas na infância ou adolescência que possam ser causa de sofrimento psicológico preexistente também foram importantes, dentre elas as que mais se destacaram foram os relatos de dificuldade para conciliar os estudos com o lazer ( $p=0,001)$ e o desconforto físico no período de provas ou apresentação de seminários $(p=0,001)$ (Tabela 3).

Tabela 3. Aspectos psicossociais e associação com casos suspeitos de transtornos mentais comuns entre os estudantes de medicina da UFES, 2007 ( $N=229)$

\begin{tabular}{|c|c|c|c|c|c|}
\hline Variáveis & $\mathrm{N}$ & $\%$ & \% TMC & $p$ & $O R_{b}(I C 95 \%)$ \\
\hline Dificuldade para fazer amigos & & & & 0,003 & \\
\hline Sim & 56 & 24,5 & 53,6 & & $1,7(1,23-2,37)$ \\
\hline Não & 172 & 75,1 & 31,4 & & 1 \\
\hline $\begin{array}{l}\text { Sentimento de rejeição por amigos ou } \\
\text { outros da mesma faixa etária }\end{array}$ & & & & 0,029 & \\
\hline $\operatorname{Sim}$ & 16 & 7,0 & 62,5 & & $1,8(1,17-2,70)$ \\
\hline Não & 213 & 93 & 35,2 & & 1 \\
\hline $\begin{array}{l}\text { Recebimento do apoio emocional } \\
\text { necessário }\end{array}$ & & & & 0,001 & \\
\hline Não & 75 & 32,8 & 64,0 & & $2,7(1,92-3,70)$ \\
\hline Sim & 154 & 67,2 & 24,0 & & 1 \\
\hline $\begin{array}{l}\text { História de tratamento psiquiátrico } \\
\text { medicamentoso }\end{array}$ & & & & 0,020 & \\
\hline Sim & 35 & 15,3 & 54,3 & & $1,6(1,12-2,32)$ \\
\hline Não & 193 & 84,3 & 33,7 & & 1 \\
\hline História de tratamento psicoterapêutico & & & & 0,020 & \\
\hline Sim & 73 & 31,9 & 47,9 & & $1,5(1,08-2,08)$ \\
\hline Não & 156 & 68,1 & 32,1 & & 1 \\
\hline \multicolumn{6}{|l|}{$\begin{array}{l}\text { Situaçōes identificadas durante a } \\
\text { infância ou a adolescência }\end{array}$} \\
\hline Dificuldade para fazer amigos & 53 & 23,1 & 54,7 & 0,002 & $1,7(1,24-2,38)$ \\
\hline $\begin{array}{l}\text { Dificuldade para tirar dúvidas em } \\
\text { sala de aula, por timidez }\end{array}$ & 82 & 35,8 & 48,8 & 0,006 & $1,6(1,15-2,22)$ \\
\hline $\begin{array}{l}\text { Presença de medos que } \\
\text { prejudicaram o rendimento } \\
\text { escolar ou o relacionamento com } \\
\text { os colegas }\end{array}$ & 34 & 14,8 & 58,8 & 0,005 & $1,8(1,25-2,49)$ \\
\hline $\begin{array}{l}\text { Dificuldades frequentes para } \\
\text { dormir }\end{array}$ & 36 & 15,7 & 55,6 & 0,013 & $1,7(1,16-2,35)$ \\
\hline $\begin{array}{l}\text { Desconforto físico (náuseas, } \\
\text { diarreia, suor excessivo, falta } \\
\text { de apetite etc.) relacionado às } \\
\text { provas ou à apresentação de } \\
\text { seminários, trabalhos }\end{array}$ & 79 & 34,5 & 51,9 & 0,001 & $1,8(1,28-2,45)$ \\
\hline $\begin{array}{l}\text { Dificuldade de relacionamento } \\
\text { com os pais com repercussão no } \\
\text { seu dia a dia }\end{array}$ & 21 & 9,2 & 57,1 & 0,046 & $1,6(1,08-2,46)$ \\
\hline $\begin{array}{l}\text { Dificuldade para conciliar os } \\
\text { estudos com lazer ou outras } \\
\text { atividades }\end{array}$ & 72 & 31,4 & 54,2 & 0,001 & $1,9(1,34-2,55)$ \\
\hline $\begin{array}{l}\text { Identificar pelo menos uma } \\
\text { dessas situaçōes }\end{array}$ & 176 & 76,9 & 43,2 & 0,001 & $2,5(1,37-4,72)$ \\
\hline
\end{tabular}

O modelo final de regressão, apresentado na tabela 4, demonstrou associação entre TMC e "não receber o apoio emocional necessário" [OR = 7,4 (IC 95\%, 3,1-17,9)] e relatar "dificuldade para tirar dúvidas em sala de aula, por timidez", durante a infância ou adolescência [OR =2,5 (IC 95\%, 1,0-6,1)].

Tabela 4. Fatores associados aos casos suspeitos de transtornos mentais comuns entre os estudantes de medicina da UFES, 2007, no modelo final de regressão logística

\begin{tabular}{lll}
\hline Variável & $\mathbf{O R}_{A}(I C 95 \%)$ & $\mathbf{P}_{A}$ \\
\hline Não receber o apoio emocional necessário & $7,4(3,06-17,90)$ & $<0,001$ \\
$\begin{array}{l}\text { Dificuldade para tirar dúvidas em sala de aula, por timidez } \\
\text { Motivo da sobrecarga de atividades }\end{array}$ & $2,5(1,00-6,10)$ & 0,049 \\
$\begin{array}{l}\text { Cobrança pessoal } \\
\text { Pressão social da profissão }\end{array}$ & $0,4(0,15-1,01)$ & 0,051 \\
$\begin{array}{l}\text { Contribuição de carga horária de atividades como fonte de } \\
\text { tensão no curso }\end{array}$ & $2,5(0,98-6,31)$ & 0,055 \\
\hline
\end{tabular}

Variáveis no modelo: idade, sexo, religiăo, renda própria, satisfaçăo com a escolha profissional, carga horária curricular, atividades extracurriculares, motivos de sobrecarga, atividades de lazer na frequência desejada, dificuldade para fazer amigos, sentimento de rejeição, apoio emocional, tratamento medicamentoso, tratamento psicoterapêutico, situaçōes especiais na infância e adolescência.

OR (IC 95\%): odds ratio ajustado (intervalo de confiança de 95\%). A porcentagem geral dos dados utilizados na análise foi de 87.5; teste de Hosmer e Lomeshow 0,630.

\section{DISCUSSÃO}

Embora as taxas de prevalência de TMC identificadas entre os estudantes de medicina sejam extremamente variáveis, a prevalência encontrada neste estudo $(37,1 \%)$ foi comparável a de outros estudos brasileiros, permanecendo dentro do intervalo de valores já apresentados. A prevalência foi superior àquelas encontradas na Universidade Federal de Santa Maria (31,7\%), na Universidade Federal do Rio Grande do Sul (22,19\%) e na Universidade Federal da Bahia $(29,6 \%)^{3,11,18}$, mas inferior às encontradas na Universidade Federal de Pernambuco $(42,6 \%)$ e na Universidade Estadual Paulista em Botucatu $(44,7 \%)^{9,19}$.

Há relatos na literatura de associação entre sexo feminino e casos suspeitos de TMC na população geral ${ }^{6}$, porém sexo não foi uma variável estatisticamente significativa neste estudo, como também ocorreu em outros estudos entre estudantes de medicina ${ }^{3,9,19}$. Não houve associação entre condições sociodemográficas dos estudantes de medicina e TMC. A explicação possível é que, por tratar-se de um grupo muito homogêneo em suas características sociodemográficas e econômicas, as pequenas diferenças encontradas perderam sua importância diante de outras categorias mais discrepantes no grupo ${ }^{19}$.

Este estudo apontou maior prevalência de TMC entre os alunos do curso básico. Após o entusiasmo inicial da conquista de uma vaga no curso mais disputado no vestibular e a entrada na universidade, os alunos se deparam com uma fase de frustração, causada pela mudança de hábitos do cotidiano, dificuldade na administração do tempo entre uma excessiva carga de estudos e pouco tempo para atividades de lazer ${ }^{20}$. Nos resultados estratificados por ano do curso, as maiores frequências foram encontradas no segundo e no quarto anos, 
que são momentos de transição para os estágios seguintes do curso. Não há consenso na literatura sobre o momento do curso no qual o risco de desenvolver transtornos mentais é maior, pois esse dado sofre influência das características de cada escola médica, das disciplinas, dos professores e dos alunos envolvidos, o que torna complexa a comparação com outros estudos ${ }^{3,14,19}$. No último ano, mesmo com a proximidade do fim da graduação, das provas de residência e da inserção no mercado de trabalho, encontrou-se a menor frequência de casos suspeitos de TMC de todo o curso.

Estudos transversais têm como limitação a impossibilidade de atribuir causalidade ou consequência às associações encontradas, já que analisam desfecho e exposição simultaneamente. Entretanto, apontam as direções nas quais os fatores preditivos se associam com o desfecho estudado. Para minimizar as dificuldades na identificação da sequência temporal dos eventos, questões referentes a situações sugestivas de sofrimento mental preexistente, como as questões sobre situações específicas durante a infância e a adolescência, foram incluídas no questionário. Procurou-se minimizar o viés de informação com o anonimato das respostas, preenchimento dos questionários em casa e a utilização de instrumentos autoaplicáveis, apenas com respostas objetivas. Quanto a erros na classificação do TMC, buscou-se um instrumento já validado anteriormente no Brasil ${ }^{16}$, escolhendo um ponto de corte baseado em estudos anteriores com estudantes de medicina9,19. É importante ressaltar, porém, que a avaliação pode ter sido influenciada pelo momento vivido por cada estudante na ocasião, já que o questionário deveria ser respondido baseado nos últimos 30 dias. Da mesma forma, os casos suspeitos de TMC podem não ter sido detectados nos estudantes já em acompanhamento psiquiátrico ou psicológico. Por fim, deve-se considerar que o SRQ-20 simplesmente rastreia casos suspeitos de TMC, sendo o padrão-ouro para diagnóstico a entrevista com o psiquiatra ${ }^{21}$.

É importante também relatar a dificuldade no processo de seleção para os alunos dos dois últimos anos do curso, pois eles não estão mais em sala de aula. Entretanto, como estavam sendo selecionados 50\% dos alunos de cada período, não se acredita que esse fato tenha invalidado os resultados. Estudos longitudinais sobre morbidade psiquiátrica seriam mais adequados para estimar a ocorrência de transtornos mentais durante a vida acadêmica e a influência do currículo médico nesse contexto, pois poderiam contribuir para a identificação de fatores de risco potenciais no desenvolvimento de TMC nessa população?.

Diversos são os fatores preditivos para TMC relatados na literatura, como exemplo, sentir-se sobrecarregado, presença de situações especiais durante a infância e adolescência que indiquem sofrimento mental preexistente, alterações no padrão de sono, avaliação ruim sobre desempenho escolar, dificuldade para fazer amigos, pensar em abandonar o curso e não receber o apoio emocional de que necessita foram identificados na literatura como alguns dos fatores de maior risco para a ocorrência de TMC 3,9,19,22,23. O presente trabalho também demonstrou que "não receber o apoio emocional necessário" foi um fator de risco para os casos suspeitos de TMC, assim como relatar "dificuldade para tirar dúvidas em sala de aula, por timidez" durante a infância ou adolescência, dado que não foi identificado em outros estudos, embora tenha sido questionado?.

As variáveis associadas aos casos suspeitos de TMC no modelo final de regressão pertencem ao bloco de aspectos psicossociais dos estudantes, enquanto as características socioeconômicas e acadêmicas não se mostraram significativas no desenvolvimento de TMC. Essa observação foi corroborada por vários estudos, entre estudantes de medicina e médicos, que demonstraram que a maior vulnerabilidade desses aos transtornos mentais estava fortemente associada à adaptação na vida anterior à escola médica ${ }^{22,24}$.

Muitas das características envolvidas na escolha pela carreira médica predispõem os estudantes a desordens emocionais e doença mental. Em uma revisão sobre a propensão dos estudantes e médicos para distúrbios emocionais e psiquiátricos, o autor destacou o papel das experiências de vida na escolha da carreira e na determinação da vulnerabilidade às solicitações da profissão ${ }^{22}$. Para uma porcentagem dos estudantes, um dos motivos da opção profissional foi uma tentativa de restaurar experiências emocionais da infância, ligadas a situações de impotência e/ou de abandono emocional. Seria, portanto, uma resposta adaptativa a uma história de fragilidade e de baixa autoestima anterior à escola médica ${ }^{24}$.

As instituições de ensino superior devem refletir criticamente sobre esse contexto do ensino médico, conhecer as características de seus alunos e os momentos considerados críticos ao longo do curso, com a finalidade de articular estratégias para auxiliar o estudante a enfrentar as dificuldades do cotidiano ${ }^{19}$. A tarefa primordial é fornecer ao aluno um espaço para reflexão sobre seus sentimentos e emoções, mediante debate aberto e franco sobre as vulnerabilidades, limitações e patologias dos estudantes, com o mesmo empenho e dedicação com que é feito em relação às suscetibilidades e patologias dos pacientes ${ }^{24}$. A importância da saúde mental é reconhecida pela OMS, desde a sua origem, o que se reflete na sua própria definição de saúde, como "um estado de completo bem-estar físico, mental e social"6.

Um ponto importante a ser enfatizado neste estudo é que as turmas entrevistadas foram as últimas a entrar na universidade pelo sistema tradicional de seleção, antes do sistema de cotas, implantado na UFES em 2008, que destinou 40\% das vagas aos estudantes oriundos de escola pública. Será importante, diante desse novo sistema, conhecer o perfil socioeconômico dessa população e avaliar se o sistema de inclusão social está alcançando seus objetivos, bem como compreender como esses alunos estão se inserindo na faculdade, avaliar o 
seu desempenho acadêmico e a resposta aos estressores do curso e expectativas em relação à graduação e à profissão.

\section{CONCLUSÃO}

Os resultados encontrados demonstram que, nessa população, as queixas psicossociais são as mais associadas aos quadros de TMC e que a alta prevalência encontrada pode estar associada a fatores presentes desde antes da graduação, que podem estar relacionados até mesmo aos motivos que levam esses alunos a escolherem a carreira médica. Essas informações são importantes para subsidiar ações para prevenção e cuidado com a saúde mental dos estudantes de medicina, melhorando a qualidade de vida deles e auxiliando em sua formação profissional.

\section{CONFLITO DE INTERESSE}

Os autores declaram não existir conflito de interesse.

\section{REFERÊNCIAS}

1. Tófoli LFF. Transtornos somatoformes, síndromes funcionais e sintomas físicos sem explicação. In: Lopes AC, organizador. Tratado de clínica médica. São Paulo: Roca; 2006, p. 2507.

2. Goldberg D, Huxley P. Commom mental disorders: a bio-social model. London: Tavistock; 1992.

3. Almeida AM, Godinho TM, Bitencourt AG, et al. Common mental disorders among medical students. J Bras Psiquiatr. 2007;56(4):245-51.

4. Murray (J, Lopez AD. Alternative projections of mortality and disability by cause 19902020: Global Burden of Disease Study. Lancet. 1997;349:1498-504.

5. Bijl RV, Ravelli A, Van Zessen G. Prevalence of psychiatric disorder in the general population: results of The Netherlands Mental Health Survey and Incidence Study (NEMESIS). Soc Psychiatry Psychiatr Epidemiol. 1998;33:587-95.

6. Organização Mundial da Saúde (OMS). Relatório mundial da saúde. Saúde mental: nova concepção, nova esperança. Lisboa; 2001.
7. Polydoro SAJ, Primi R, Serpa MNF, Zaroni MMH, Pombal KCP. Desenvolvimento de uma escala de integração ao ensino superior. Psico-USF. 2001;6:11-7.

8. Mowbray CT, Megivern D, Mandiberg JM, Strauss S, Stein CH, Collins K, et al. Campus mental health services: recommendations for change. J Orthopsychiatry. 2006;76(2):226-37.

9. Facundes VLD, Ludermir AB. Common mental disorders among health care students. Rev Bras Psiquiatr. 2005:27:194-200.

10. Firth J. Levels and sources of stress in medical students. Br Med J. 1986;292:1177-80.

11. Benvegnu LA, Deitos F, Copette FR. Problemas psiquiátricos menores em estudantes de Medicina da Universidade Federal de Santa Maria, RS, Brasil. Rev Psiquiatr Rio Gd Sul. 1996:18:229-33.

12. Vaz RF, Mbajiorgu EF, Acuda SW. A preliminary study of stress levels among first year medical students at the University of Zimbabwe. Cent Afr J Med. 1998;44:214-9.

13. Roberts LW, Warner TD, Liketsos C, Frank E, Ganzini L, Carter D. Perceptions of academic vulnerability associated with personal illness: a study of 1,027 students at nine medical schools. Colllaborative Research Groups on Medical Student Health. Compr Psychiatry. 2001:42(1):1-15.

14. Rosal MC, Ockene IS, Ockene JK, Barret SV, Ma Y, Hebert JR. A longitudinal study of students depression at one medical school. Acad Med. 1997;72:542-6.

15. Harding TW, Arango MV, Baltazar J, Climent CE, Ibrahim HHA, Ladrido-Inacio L, et al. Mental disorders in primary health care: a study of the frequency and diagnosis in four developing countries. Psychol Med. 1980;10:231-41.

16. Mari JJ, Williams PA. A validity study of a Psychiatric Screening Questionnaire (SRQ 20) in primary care in city of São Paulo. Br J Psychiatry. 1986;148:23-6.

17. World Health Organization. A user's guide to the Self Reporting Questionnaire (SRQ). Geneva: Division of Mental Health; 1994.

18. Loayza HMP, Ponte TS, Carvalho CG, Pedrotti MR, Nunes PV, Souza CM, et al. Association between mental health screening by self-report questionnaire and insomnia in medical students. Arq Neuropsiquiatr. 2001;59(2-A):180-5.

19. Lima MC, Domingues MS, Cerqueira AT. Prevalence and risk factors of common mental disorders among medical students. Rev Saude Publica. 2006;40:1035-41.

20. Rezende CHA, Abrão CB, Coelho EP, Passos LBS. Prevalência de sintomas depressivos entre estudantes de medicina da Universidade Federal de Uberlândia. Rev Bras Educ Med. 2008; 32(3):315-23.

21. Costa AG, Ludermir AB. Transtornos mentais comuns e apoio social: estudo em comunidade rural da Zona da Mata de Pernambuco, Brasil. Cad Saude Publica. 2005;21(1):73-9.

22. Johnson WDK. Predisposition to emotional distress and psychiatric illness amongst doctors: the role of unconscious and experimental factors. Br J Med Psychol. 1991;64:317-29.

23. Ludermir AB, Melo-Filho DA. Living conditions and occupational organization associated with common mental disorders. Rev Saude Publica. 2002;36:213-21.

24. Millan LR, Arruda PCV. Assistência psicológica ao estudante de medicina: 21 anos de experiência. Rev Assoc Med Bras. 2008;54(1):90-4 